\title{
@creative
}

Also available at http://amc-journal.eu

ISSN 1855-3966 (printed edn.), ISSN 1855-3974 (electronic edn.)

ARS MATHEMATICA CONTEMPORANEA 14 (2018) 39-54

\section{Game distinguishing numbers of Cartesian products}

\author{
Sylvain Gravier \\ Institut Fourier, UMR 5582, S.F.R. Maths à Modeler, Université Grenoble Alpes \\ 100 , rue des maths BP74 \\ 38402 Saint-Martin-d'Hères Cedex, France \\ Kahina Meslem \\ LaROMaD, Faculty of Mathematics, S.F.R. Maths à Modeler, U.S.T.H.B. \\ El Alia BP 32 Bab Ezzouar \\ 16111 Alger, Algeria \\ Simon Schmidt \\ Institut Fourier, UMR 5582, S.F.R. Maths à Modeler, Université Grenoble Alpes \\ 100, rue des maths BP74 \\ 38402 Saint-Martin-d'Hères Cedex, France \\ Souad Slimani \\ LaROMaD, Faculty of Mathematics, S.F.R. Maths à Modeler, U.S.T.H.B. \\ El Alia BP 32 Bab Ezzouar \\ 16111 Alger, Algeria
}

Received 15 August 2016, accepted 24 February 2017, published online 14 April 2017

\begin{abstract}
The distinguishing number of a graph $H$ is a symmetry related graph invariant whose study started two decades ago. The distinguishing number $D(H)$ is the least integer $d$ such that $H$ has a distinguishing $d$-coloring. A distinguishing $d$-coloring is a coloring $c: V(H) \rightarrow\{1, \ldots, d\}$ invariant only under the trivial automorphism. In this paper, we continue the study of a game variant of this parameter, recently introduced. The distinguishing game is a game with two players, Gentle and Rascal, with antagonistic goals. This game is played on a graph $H$ with a fixed set of $d \in \mathbb{N}$ colors. Alternately, the two players choose a vertex of $H$ and color it with one of the $d$ colors. The game ends when all the vertices have been colored. Then Gentle wins if the $d$-coloring is distinguishing and Rascal wins otherwise. This game defines two new invariants, which are the minimum numbers of colors needed to ensure that Gentle has a winning strategy, depending who starts the game. The invariant could be infinite. In this paper, we focus on the Cartesian


product, a graph operation well studied in the classical case. We give sufficient conditions on the order of two connected factors $H$ and $F$ that are relatively prime, which ensure that one of the game distinguishing numbers of the Cartesian product $H \square F$ is finite. If $H$ is a so-called involutive graph, we give an upper bound of order $D^{2}(H)$ for one of the game distinguishing numbers of $H \square F$. Finally, using in part the previous result, we compute the exact value of these invariants for Cartesian products of relatively prime cycles. It turns out that the value is either infinite or equal to 2 , depending on the parity of the product order.

Keywords: Distinguishing number, graph automorphism, combinatorial game.

Math. Subj. Class.: 05C57, 05C69, 91 A43

\section{Introduction}

In this paper, we consider only simple graphs. For a graph $H$, the sets $V(H)$ and $E(H)$ respectively denote the vertex set and the edge set of $H$. For an integer $n \geq 3$, we denote by $C_{n}$ the cycle of order $n$. For $n \geq 2$, we respectively write $K_{n}$ and $P_{n}$ for the clique and the path of order $n$. The distinguishing number $D(H)$ of a graph $H$ is a symmetry related graph invariant introduced two decades ago [1]. More precisely, $D(H)$ is the least integer $d$ such that $H$ has a distinguishing $d$-coloring. A distinguishing $d$-coloring is a vertex-coloring $c: V(H) \rightarrow\{1, \ldots, d\}$, invariant only under the trivial automorphism. More generally, we say that an automorphism $\sigma$ of $H$ preserves the coloring $c$, or is a color preserving automorphism, if for all $u \in V(H)$, we have $c(u)=c(\sigma(u))$. The automorphism group of $H$ will be denoted by $\operatorname{Aut}(H)$. Clearly, for each coloring $c$ of the vertex set of $H$, the set $\operatorname{Aut}_{c}(H)=\{\sigma \in \operatorname{Aut}(H): c \circ \sigma=c\}$ is a subgroup of $\operatorname{Aut}(H)$. A coloring $c$ is distinguishing if $\operatorname{Aut}_{c}(H)$ is trivial. The ten last years have seen a flourishing number of works on this subject, and Cartesian products of graphs were thoroughly investigated in $[2,5,7,8,12,13,15,16]$. In particular, the exact value of $D\left(K_{n} \square K_{m}\right)$ is given in [7, 13]. Another interesting result for our purpose is that if $k \geq 2$, then $D\left(C_{n_{1}} \square \cdots \square C_{n_{k}}\right)=2$, except for $C_{3} \square C_{3}$. In that case, $D\left(C_{3} \square C_{3}\right)=3$. This result is an easy consequence of more general results in [13]. Cartesian products have also been investigated in the context of distinguishing edge-coloring $[4,10]$. In this paper, we are interested in a game variant of the distinguishing number, introduced in [11]. Defining game invariants for graphs is not a new idea. The two most known game invariants are the game chromatic number, introduced by Brahms [6] in 1981, and the game domination numbers introduced more recently by Brešar, Klavžar and Rall [3].

The distinguishing game is a game with two players, Gentle and Rascal, with antagonistic goals. This game is played on a graph $H$ with a fixed set of $d$ colors, $d \in \mathbb{N}$. Alternately, the two players choose a vertex of $H$ and color it with one of the $d$ colors. The game ends when all the vertices have been colored. If the $d$-coloring is distinguishing then Gentle wins. Otherwise Rascal wins.

This game defines two invariants for a graph $H$. The $\mathcal{G}$-game distinguishing number $D_{\mathcal{G}}(H)$ is the minimum number of colors needed to ensure that Gentle has a winning strategy for the game on $H$, assuming he is playing first. If Rascal is sure to win whatever the number of colors we allow, then $D_{\mathcal{G}}(H)=\infty$. Similarly, the $\mathcal{R}$-game distinguishing

E-mail addresses: sylvain.gravier@univ-grenoble-alpes.fr (Sylvain Gravier), kmeslem@usthb.dz (Kahina Meslem), simon.schmidt@univ-grenoble-alpes.fr (Simon Schmidt), sslimani@usthb.dz (Souad Slimani) 
number $D_{\mathcal{R}}(H)$ is the minimum number of colors needed to ensure that Gentle has a winning strategy, assuming Rascal is playing first. Characterizing graphs with infinite game distinguishing number seems to be a challenging open question. In [11], the authors give sufficient conditions for a graph to have one infinite game distinguishing number.

Proposition 1.1. [11] Let $H$ be a graph and $\sigma$ a nontrivial automorphism of $H$ such that $\sigma \circ \sigma=\mathrm{id}_{H}$.

1. If $|V(H)|$ is even, then $D_{\mathcal{G}}(H)=\infty$.

2. If $|V(H)|$ is odd, then $D_{\mathcal{R}}(H)=\infty$.

Also in [11], the exact values of those invariants have been computed for many cycles and hypercubes. For a large class of graphs, the so-called involutive graphs, a quadratic upper bound involving the classical distinguishing number has been provided (see Section 4 for the definition). We give here the precise statement of the results used in this paper.

Theorem 1.2. [11] Let $C_{n}$ be a cycle of order $n \geq 3$.

1. If $n$ is even (resp. odd), then $D_{\mathcal{G}}\left(C_{n}\right)=\infty$ (resp. $\left.D_{\mathcal{R}}\left(C_{n}\right)=\infty\right)$.

2. If $n$ is even and $n \geq 8$, then $D_{\mathcal{R}}\left(C_{n}\right)=2$.

3. If $n$ is odd, not prime and $n \geq 9$, then $D_{\mathcal{G}}\left(C_{n}\right)=2$.

4. If $n$ is prime and $n \geq 5$, then $D_{\mathcal{G}}\left(C_{n}\right) \leq 3$.

Moreover $D_{\mathcal{R}}\left(C_{4}\right)=D_{\mathcal{R}}\left(C_{6}\right)=3, D_{\mathcal{G}}\left(C_{3}\right)=\infty$ and $D_{\mathcal{G}}\left(C_{5}\right)=D_{\mathcal{G}}\left(C_{7}\right)=3$.

Theorem 1.3. [11] If $H$ is an involutive graph with $D(H) \geq 2$, then $D_{\mathcal{R}}(H) \leq D^{2}(H)+$ $D(H)-2$.

In this paper, we deal with Cartesian products of connected relatively prime graphs. In Section 3, we prove the following theorem which gives sufficient conditions on the order of the two factors to have a finite distinguishing number.

Theorem 1.4. Let $H$ and $F$ be two nontrivial connected relatively prime graphs of order $n$ and $m$, respectively.

1. If $n$ is even and $m \geq n-1$, then $D_{\mathcal{R}}(H \square F) \leq m+1$.

2. If $n$ is odd, $m$ is even and $m \geq 2 n-2$, then $D_{\mathcal{R}}(H \square F) \leq m+1$.

3. If $n$ and $m$ are odd and $m \geq 2 n-1$, then $D_{\mathcal{G}}(H \square F) \leq m+1$.

In Section 4, we investigate the case where one factor is an involutive graph. In that case, if the classical distinguishing number of the other factor is not too big, we have a quadratic upper bound involving the classical distinguishing number of the involutive factor.

Theorem 1.5. Let $H$ be a connected involutive graph of order $n$, with $D(H) \geq 2$ and $F$ a connected graph, relatively prime to $H$. If $D(F) \leq\left(\begin{array}{c}\frac{n+d^{2}+d}{2}-1 \\ \frac{d^{2}+d}{2}-1\end{array}\right)$, then $D_{\mathcal{R}}(H \square F) \leq$ $d^{2}+d-2$, where $d=D(H)$. 
Finally, in Section 5, we compute the exact value of the two invariants for Cartesian products of relatively prime cycles. Since even cycles are involutive graphs, a part of this result arises as a corollary of the above theorem.

Theorem 1.6. Let $n_{1}, \ldots, n_{k}$, with $k \geq 2$, be $k$ distinct natural numbers greater or equal to 3 .

1. If $\prod_{i=1}^{k} n_{i}$ is even, then $D_{\mathcal{G}}\left(C_{n_{1}} \square \cdots \square C_{n_{k}}\right)=\infty$ and $D_{\mathcal{R}}\left(C_{n_{1}} \square \cdots \square C_{n_{k}}\right)=2$.
2. If $\prod_{i=1}^{k} n_{i}$ is odd, then $D_{\mathcal{R}}\left(C_{n_{1}} \square \cdots \square C_{n_{k}}\right)=\infty$ and $D_{\mathcal{G}}\left(C_{n_{1}} \square \cdots \square C_{n_{k}}\right)=2$.

All these three results highly involve the so-called fiber-strategy for Gentle. Section 2 is devoted to the definition and the properties of this strategy.

\section{Cartesian products of graphs and the fiber-strategy}

In this section, we give the minimal background needed on Cartesian products and define an efficient strategy for Gentle, the so-called fiber-strategy, based on the fibers structure of Cartesian products of graphs. For more information on Cartesian product see [14].

\subsection{Cartesian products of graphs}

Let $H$ and $F$ be two connected relatively prime graphs. The vertices of $H \square F$ will be denoted by $(u, v)$, where $u \in V(H)$ and $v \in V(F)$. An $H$-fiber is a subgraph of $H \square F$ induced by all the vertices having the same second coordinate. We write $H^{v}$, where $v \in$ $V(F)$, for the $H$-fiber induced by $\{(u, v) \mid u \in V(H)\}$. Similarly, we define $F^{u}$, with $u \in V(H)$. The $H$-fibers and the $F$-fibers are respectively isomorphic to $H$ and $F$. The automorphism group of $H \square F$ is isomorphic to $\operatorname{Aut}(H) \times \operatorname{Aut}(F)$. If $\sigma$ is an automorphism of $H \square F$, it can be seen as a couple $(\psi, \phi)$, where $\psi \in \operatorname{Aut}(H)$ and $\phi \in \operatorname{Aut}(F)$. In that case, $\sigma((u, v))=(\psi(u), \phi(v))$. Another important fact is that $\sigma$ must send an $H$-fiber to another $H$-fiber and the same for the $F$-fibers. More precisely, $\sigma\left(H^{v}\right)=H^{\phi(v)}$ and $\sigma\left(F^{u}\right)=F^{\psi(u)}$. To show that a color preserving automorphism has to be the identity of $\operatorname{Aut}(H \square F)$, we will mostly proceed as follows. First, we show that an $H$-fiber cannot be sent to another one, which means that $\phi$ is the identity and $\sigma$ fixes the $H$-fibers setwise. Using these informations, we prove that $\psi$ is also the identity.

\subsection{Fiber-strategy}

Now, we state some technical results about the fiber-strategy, a strategy that Gentle will follow in almost all the proofs of our main results. In a game on $H \square F$, with $H$ nontrivial, we say that Gentle follows the $H$-fiber-strategy if we are in one of the following two cases.

Case 0:

- $|V(H)|$ is even and Rascal starts.

- When Rascal plays in an H-fiber, Gentle plays in the same H-fiber.

Case 1: 
- $|V(H)|$ is odd.

- $|V(F)|$ is even and Rascal starts or $|V(F)|$ is odd and Gentle starts.

- When Rascal colors the first vertex of a totally uncolored H-fiber, Gentle colors the first vertex of another such $H$-fiber.

- When Rascal colors a vertex in an $H$-fiber which already has a colored vertex, Gentle colors a vertex in the same $H$-fiber.

The $H$-fiber strategy is always valid. In Case 0 , the parity of each $H$-fiber ensures that Rascal will always be the first to run out of moves in an $H$-fiber. Hence, Rascal is always the first to play in each $H$-fiber. In Case 1 , after Gentle's move, there is always an even number of remaining totally uncolored $H$-fibers. Hence, Rascal will always be the first to run out of new totally uncolored $H$-fibers to play in.

The following properties are easy and given without proof. They are however fundamental to prove the results in the further sections.

Proposition 2.1. Assume that Gentle plays according to the H-fiber-strategy.

1. He will color the last vertex of each $H$-fiber.

2. In Case 0, Rascal will be the first to play in all the $H$-fibers. Then, Gentle will play all the second moves in each $H$-fiber, Rascal will play all the third moves and so on.

3. In Case 1, Gentle will play the first in exactly $\left[\frac{|V(H)|}{2}\right\rceil$ different $H$-fibers. Then, Rascal will play all the second moves in each H-fiber, Gentle will play all the third moves and so on.

In Case 0, the moves in an $H$-fiber alternate exactly as in the game played only on $H$, when Rascal starts (see Fig. 1, where $R_{i}$ and $G_{i}$ respectively denote the $i$-th move of Rascal and Gentle). This property will be often used by Gentle to play in an $H$-fiber following a winning strategy for the game on $H$. In Case 1 , in an $H$-fiber where Gentle plays first, the moves alternate as in the game played only on $H$, when Gentle starts. In an $H$-fiber where Rascal plays first, the only difference is that he is going to play the two first moves in a row (see Fig. 2, where $R_{i}$ and $G_{i}$ have the same meaning as before). In that case the Lemma 2.2 could be useful. It says that for vertex transitive graphs, if you can win the game playing first, then you can be a real gentleman and let your opponent play this first move.

Lemma 2.2. Assume $H$ is vertex transitive. Then either all the first moves are winning for the first player or they are all losing.

Proof. Assume there is $u_{0} \in V(H)$ such that coloring $u_{0}$ with 1 is a winning move for the first player. We have to prove that for any $v \in V(H)$, coloring $v$ with 1 is also a winning move. Let $G$ be the game in which the first player has played the winning move $u_{0}$ and let $G^{\prime}$ be the game in which his first move has been to color another vertex $v_{0}$ with 1 . Let $c$ and $c^{\prime}$ be respectively the coloring built during the game $G$ and $G^{\prime}$. Since $H$ is vertex transitive, there exists $\sigma \in \operatorname{Aut}(H)$ such that $\sigma\left(v_{0}\right)=u_{0}$. The winning strategy for the first player in $G^{\prime}$ is defined by his winning strategy in $G$. When his opponent colors a vertex $w$ in the game $G^{\prime}$, the first player imagines that his opponent has colored $\sigma(w)$ in $G$ with the same color. There is a vertex $w^{\prime}$ such that coloring $w^{\prime}$ is a winning answer for the 


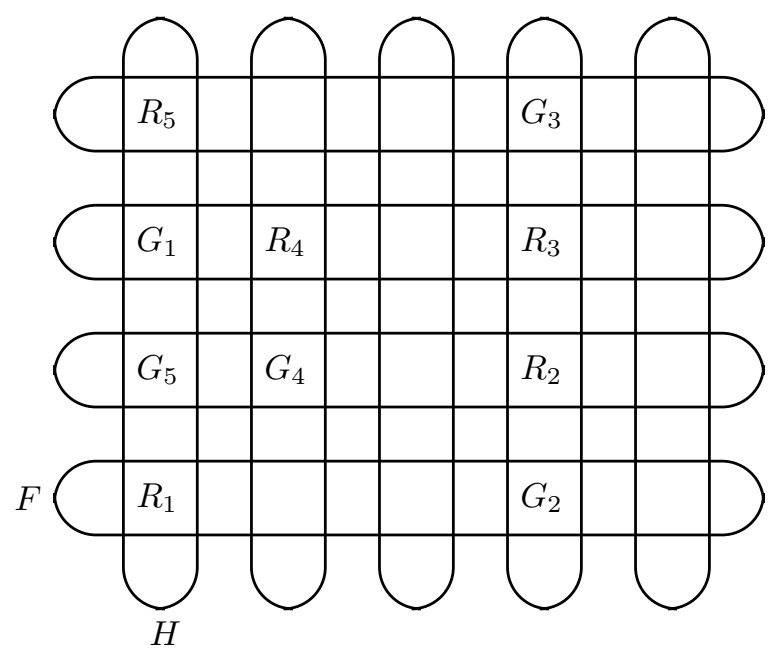

Figure 1: How moves alternate in Case 0 of the $H$-fiber strategy (Rascal starts).

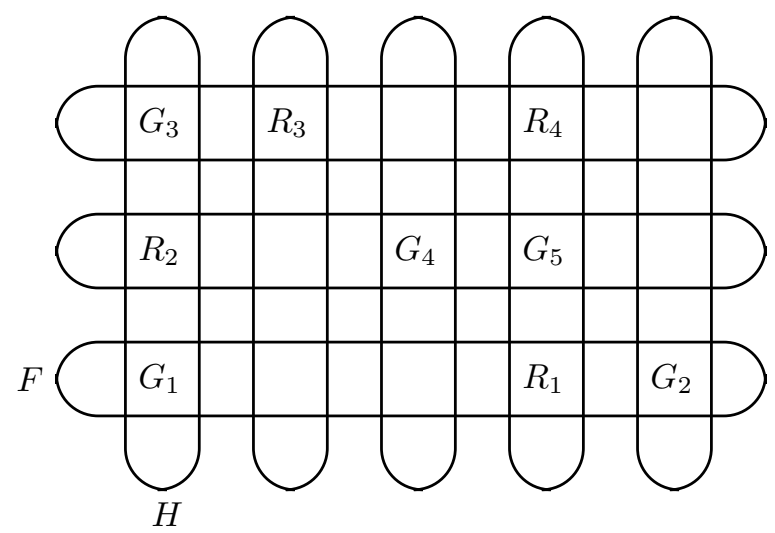

Figure 2: How moves alternate in Case 1 of the $H$-fiber strategy (Gentle starts).

first player in $G$. In the game $G^{\prime}$, the first player's answer will be to color $\sigma^{-1}\left(w^{\prime}\right)$ such that $c^{\prime}\left(\sigma^{-1}\left(w^{\prime}\right)\right)=c\left(w^{\prime}\right)$.

By assumption, the coloring $c$ is a winning one for the first player. Moreover, for any $v \in V(H), c(v)=c^{\prime}(\sigma(v))$. Hence, an automorphism $\psi$ preserves the coloring $c$ if and only if $\sigma \circ \psi \circ \sigma^{-1}$ preserves the coloring $c^{\prime}$. This shows that $c^{\prime}$ is also a winning coloring for the first player. In conclusion, if there is a winning move for the first player, then any first move is a winning move for him. 


\section{Cartesian products of complete graphs.}

Our goal in this section is to prove Theorem 1.4 which asserts, under certain conditions on their orders, that for two nontrivial connected graphs $H$ and $F$ that are relatively prime, and of respective order $n$ and $m$, at least one game distinguishing number of $H \square F$ is finite. Except when both cardinalities are equal, it comes directly from the following theorem involving Cartesian products of complete graphs. Indeed, a distinguishing coloring of $K_{n} \square K_{m}$ is always a distinguishing coloring of $H \square F$. In the first item of Theorem 1.4, when both factors have the same even cardinality, the corresponding product of complete graphs is not covered by the below result. But, we are in fact going to prove that Gentle's strategy breaks all automorphisms of the subgroup of $\operatorname{Aut}\left(K_{n} \square K_{m}\right)$ isomorphic to $\operatorname{Aut}\left(K_{n}\right) \times \operatorname{Aut}\left(K_{m}\right)$. A coloring which distinguishes this subgroup will always be a distinguishing coloring of $H \square F$, when the factors are relatively prime.

Theorem 3.1. Let $n$ and $m$ be two distinct natural numbers greater or equal to 2 .

1. If $n \times m$ is even (resp. odd), then $D_{\mathcal{G}}\left(K_{n} \square K_{m}\right)=\infty\left(\right.$ resp. $\left.D_{\mathcal{R}}\left(K_{n} \square K_{m}\right)=\infty\right)$.

2. If $n$ is even, $m \neq n$ and $m \geq n-1$, then $D_{\mathcal{R}}\left(K_{n} \square K_{m}\right) \leq m+1$.

3. If $n$ is odd, $m$ is even and $m \geq 2 n-2$, then $D_{\mathcal{R}}\left(K_{n} \square K_{m}\right) \leq m+1$.

4. If $n$ and $m$ are odd and $m \geq 2 n-1$, then $D_{\mathcal{G}}\left(K_{n} \square K_{m}\right) \leq m+1$.

Proof. The first item is a straightforward application of Proposition 1.1. For the last items, note that $n \neq m$. Hence, the two factors $K_{n}$ and $K_{m}$ are relatively prime. The vertices of $K_{n} \square K_{m}$ are denoted by $(i, j)$, with $i \in\{1, \ldots, n\}$ and $j \in\{1, \ldots, m\}$. The meta-color of a $K_{n}$-fiber is the list $\left(c_{1}, \ldots, c_{m+1}\right)$, where $c_{l}$, with $l \in\{1, \ldots, m+1\}$, is the number of vertices in this fiber which are colored with the color $l$ at the end of the game. An important remark is that a color preserving automorphism also preserves the meta-colors of the $K_{n}$-fibers.

We are going to prove the second statement. We have to give a winning strategy for Gentle playing second with $m+1$ colors. A proper edge coloring of $K_{n}$, with $n-1$ colors gives $n-1$ perfect matchings, whose union covers all the edges of $K_{n}$. We denote these matchings by $M_{1}, \ldots, M_{n-1}$. Gentle's winning strategy is as follows. First of all, he plays according to the $K_{n}$-fiber-strategy. If Rascal plays in one of the fibers $K_{n}^{j}$, with $j \in\{1, \ldots, n-1\}$, Gentle plays with respect to the matching $M_{j}$. It means that if Rascal colors the vertex $(i, j)$, then Gentle colors the unique vertex $(k, j)$ in $K_{n}^{j}$, such that $i k$ is an edge in the matching $M_{j}$. Otherwise, he plays as he wants with respect to the $K_{n}$-fiberstrategy. See Fig. 3, where $R_{i}$ and $G_{i}$ respectively denote the $i$-th move of Rascal and Gentle. Gentle chooses the colors as follows. First, he always plays a color different from the one used by Rascal just before. Second, if he has to color the last vertex of a $K_{n}$-fiber, he chooses the color in a way that the meta-color of this fiber is distinct from all the metacolors of the already totally colored $K_{n}$-fibers. He has at most $m-1$ meta-colors to avoid. It is always possible because he can choose among the $m$ colors not used by Rascal just before.

Let us prove now that this strategy yields a distinguishing coloring $c$. Applying the above strategy, Gentle will color the last vertex of each $K_{n}$-fiber (see Proposition 2.1). Hence, he controls the meta-color of all the $K_{n}$-fibers. They will all have a distinct metacolor at the end of the game. Therefore, a color preserving automorphism $\sigma$ cannot switch 


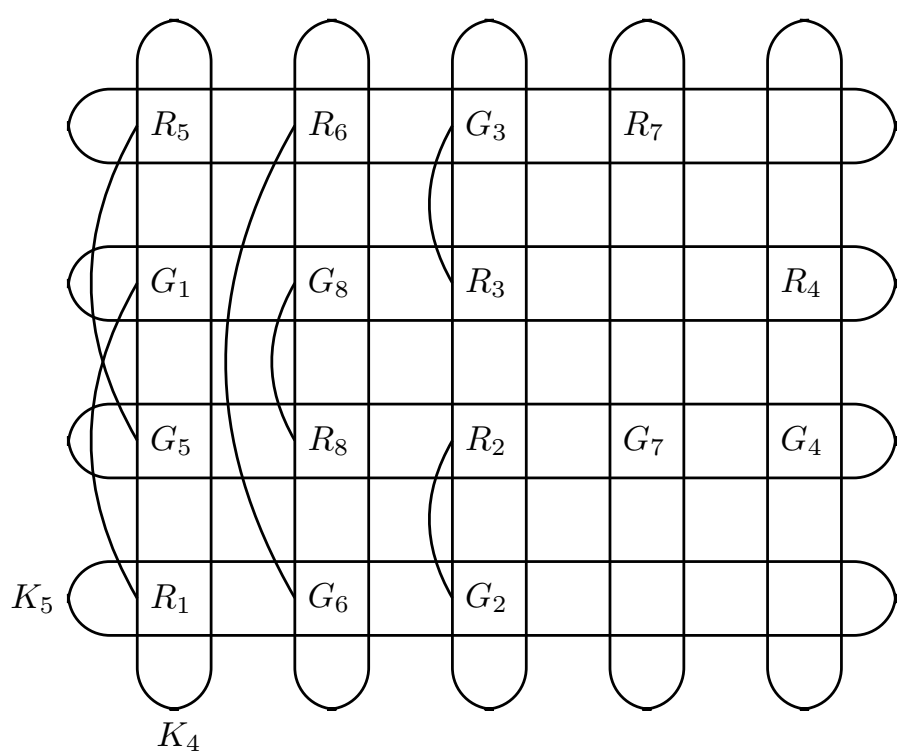

Figure 3: Playing according to the matchings in a $K_{4}$-fiber strategy.

these fibers. It means that $\sigma=\left(\psi, \mathrm{id}_{K_{m}}\right)$, where $\psi \in \operatorname{Aut}\left(K_{n}\right)$. Assume that $\psi$ is not the identity. There exists $i \in\{1, \ldots, n\}$, such that $\psi(i) \neq i$. The edge $\psi(i) i$ belongs to a matching $M_{j}$, with $j \in\{1, \ldots, n-1\}$. Since Gentle has colored either $(i, j)$ or $(\psi(i), j)$, these two vertices have not the same color. It shows that the automorphism $\left(\psi, \operatorname{id}_{K_{m}}\right)$ does not preserve the coloring $c$. In conclusion, $c$ is a distinguishing coloring.

We prove now the third item. The general ideas are similar as above, but since the $K_{n}$ fibers have odd order, a matching does not cover all the vertices of $K_{n}$. Hence, if Rascal is the first to play in a $K_{n}$-fiber, Gentle cannot immediately play with respect to a matching. Since $n$ is odd, we need $n$ matchings to cover all the edges of $K_{n}$. Each matching does not cover exactly one vertex and this uncovered vertex is distinct for each of them. We denote by $M_{j}$, with $j \in\{1, \ldots, n\}$, the matching which does not cover the vertex $j$. Without lost of generality, we assume that Rascal's first move is to color the vertex $(1,1)$ in $K_{n}^{1}$. Gentle will again follow a $K_{n}$-fiber strategy. Hence, he will be the first to color a vertex in exactly $\frac{m}{2}$ different $K_{n}$-fibers, say $K_{n}^{2}, \ldots, K_{n}^{\frac{m}{2}+1}$. When Gentle colors a vertex of $K_{n}^{j}$, with $j \in\left\{1, \ldots, \frac{m}{2}+1\right\}$, if it is the first vertex of $K_{n}^{j}$ to be colored, he chooses the vertex $(j, j)$. Otherwise, he plays with respect to the matching $M_{j}$. When the fiber-strategy leads him to play in other $K_{n}$-fibers, he plays wherever he wants with respect to the $K_{n}$-fiber strategy. For the choice of the colors, he plays as in the previous strategy. The proof that the coloring built during the game is distinguishing is exactly the same. Just note that by hypothesis, $\frac{m}{2}+1 \geq n$. Hence, Gentle has enough $K_{n}$-fibers to use the $n$ matchings needed to cover all the edges of $K_{n}$.

The proof of the last item is the same as for the previous one. Because Gentle starts, he will be the first to play in $\frac{m+1}{2}$ different $K_{n}$-fibers, which is by hypothesis greater or equal to $n$. 
For $K_{2} \square K_{m}$, we can compute the exact value of $D_{\mathcal{R}}$. For $m \in\{2,3,4\}$, it is shown in [11] that $D_{\mathcal{R}}\left(K_{2} \square K_{m}\right)=3$. For $m \geq 5$, we are going to prove that we need exactly $m$ colors. Then, the bound obtained above is close to be tight.

Let $c$ be a coloring of $K_{2} \square K_{m}$, with $m \geq 5$. We say that two distinct $K_{2}$-fibers, $K_{2}^{i}$ and $K_{2}^{j}$ are colored the same if $c\left(K_{2}^{i}\right)=c\left(K_{2}^{j}\right)$. If we have also $c((i, 1))=c((j, 1))$, we say that the two fibers are strictly colored the same.

Proposition 3.2. If $m \geq 5$, then $D_{\mathcal{R}}\left(K_{2} \square K_{m}\right)=m$.

Proof. First, we show that with $m$ distinct colors Gentle has a winning strategy. Recall that Rascal starts. When Rascal plays in a $K_{2}$-fiber, Gentle answers by coloring the second vertex of this $K_{2}$-fiber. That means he plays according to a $K_{2}$-fiber strategy. He colors in a way that the new colored $K_{2}$-fiber is not colored the same as another $K_{2}$-fiber already colored before. This is always possible, because there are at most $m-1$ different $K_{2}$-fibers colored before and Gentle can use $m$ colors. Moreover, he can ensure that at least one $K_{2}$ fiber is not monochromatic. Let us prove now that this strategy yields a distinguishing coloring. Assume $\sigma$ is a color preserving automorphism. Then $\sigma\left(K_{2}^{i}\right)=K_{2}^{i}$, for all $i \in\{1, \ldots, m\}$. But, there is at least one bi-chromatic $K_{2}$-fiber. Hence, $\sigma$ must also fix this $K_{2}$-fiber pointwise. Therefore, $\sigma$ has to fix all the $K_{2}$-fibers pointwise. In conclusion, $\sigma$ is the identity.

It remains to prove that Rascal has a winning strategy, if strictly less than $m$ colors are allowed during the game. Remark that, if two distinct $K_{2}$-fibers, $K_{2}^{i}$ and $K_{2}^{j}$ are strictly colored the same at any moment in the game, then Rascal wins. Indeed, there is an automorphism $\sigma$ such that $\sigma((i, 1))=(j, 1), \sigma((i, 2))=(j, 2)$ and $\sigma$ fixes all the other vertices.

Rascal starts by coloring $(1,1)$ with 1 . There are two cases.

Case 1: Gentle colors the vertex $(1,2)$.

Rascal answers by coloring $(2,1)$ with 1 . If Gentle colors a vertex different than $(2,2)$, Rascal wins at his next turn by coloring $(2,2)$ with the same color as $(1,2)$. So, we can suppose that Gentle colors $(2,2)$. Turn by turn, this shows that Rascal can color all the vertices of the form $(i, 1)$ with the color 1 , and that Gentle is forced to color only the vertices of the form $(i, 2)$. Since Gentle has strictly less than $m$ colors at his disposal, there are two vertices $\left(i_{0}, 2\right),\left(j_{0}, 2\right)$, which receive the same color. Hence, the two $K_{2}$-fibers, $K_{2}^{i_{0}}$ and $K_{2}^{j_{0}}$ will be strictly colored the same and Rascal will win.

Case 2: Gentle first move is to color the vertex $(2, x)$, with $x \in\{1,2\}$.

Rascal answers by coloring with 1 the vertex $(3,1)$. Now, if Gentle plays in $K_{2}^{1}$ or $K_{2}^{3}$, Rascal wins because he can play such that $K_{2}^{1}$ and $K_{2}^{3}$ are strictly colored the same. Suppose that Gentle plays a vertex which is not in $K_{2}^{1}$ or $K_{2}^{3}$. Since $m \geq 5$, at least one vertex in the fiber $K_{m}^{1}$ is still uncolored, say $(5,1)$. Rascal replies by coloring this vertex with 1 . The vertices $(1,2),(3,2)$ and $(5,2)$ are still uncolored. Rascal can ensure that at least two of the three $K_{2}$-fibers, $K_{2}^{1}, K_{2}^{3}$ and $K_{2}^{5}$ are strictly colored the same. Indeed, if Gentle is the first to color one of these three uncolored vertices, Rascal copies this color in one of the two remaining vertices. Otherwise, he will be able to decide the coloring of two of them. In conclusion, Rascal will also win in this second case.

Of course, Theorem 3.1 does not cover all possibilities. We did not manage to prove that in the remaining cases the invariants are finite. But we know that the $K_{n}$-fiber strategy used above by Gentle will fail in these cases. More precisely, we have the following proposition. 
Proposition 3.3. Let $n$ and $m$ be two distinct natural numbers at least equal to 2 . Whatever the number of colors is allowed, if Gentle follows a $K_{n}$-fiber strategy on $K_{n} \square K_{m}$, he looses in both of the following cases:

- Rascal starts, $n$ is odd, $m$ is even and $m<2 n-2$,

- Gentle starts, $n$ and $m$ are odd and $m<2 n-1$.

Proof. We prove the first statement. The second can be proved in exactly the same way. Rascal's winning strategy is to create two $K_{m}$-fibers, say $K_{m}^{1}$ and $K_{m}^{n}$, which are strictly colored the same. More precisely, if $u \in K_{m}^{1}$ and $v \in K_{m}^{n}$ are in the same $K_{n}$-fiber then they share the same color. In that case, the automorphism which only permutes $K_{m}^{1}$ and $K_{m}^{n}$ is a color preserving automorphism.

Rascal proceeds as follows. He plays his $\frac{m}{2}$ first moves in the same $K_{m}$-fiber, say $K_{m}^{1}$. Since Gentle plays according to a $K_{n}$-fiber strategy, at the end of the $\left(\frac{m}{2}\right)^{\text {th }}$ turn of Gentle each $K_{n}$-fiber has exactly one colored vertex. These $m$ first moves are called the first phase of the game. Let $k$ be the number of uncolored vertices in $K_{m}^{1}$ at the end of this first phase. We have: $0 \leq k \leq \frac{m}{2}$ ( $k$ could be equal to 0 , if Gentle has only played in $K_{m}^{1}$ during the first phase). The forthcoming $k$ moves of Rascal and $k$ moves of Gentle will be called the second phase of the game. In this phase, when Rascal plays in a $K_{n}$-fiber, Gentle has to answer by a move in this same $K_{n}$-fiber. Hence, Rascal can play all the $k$ remaining uncolored vertices of $K_{m}^{1}$. Let $u$ be such a vertex. There is a unique vertex $v$ in the same $K_{n}$-fiber than $u$, which is already colored (this vertex has been colored by Gentle during the first phase). Rascal copies the color of $v$ to color $u$. During this second part of the game, Gentle has played in at most $k$ distinct $K_{m}$-fibers. Since $m<2 n-2$, then $k<n-1$. Hence, there exists a $K_{m}$-fiber, say $K_{m}^{n}$, in which Gentle has not played during this second phase. In $K_{m}^{n}$, there is at most one colored vertex, say $w$. In that case, $w$ has been colored by Gentle during the first phase of the game. This vertex $w$ shares the same color as the vertex of $K_{m}^{1}$, which is in the same $K_{n}$-fiber (this vertex has been colored by Rascal during the second phase). Therefore, Rascal can now color all the uncolored vertices of $K_{m}^{n}$, such that $K_{m}^{1}$ and $K_{m}^{n}$ are strictly colored the same.

\section{Cartesian products of involutive graphs.}

In this section, we study the game distinguishing numbers of Cartesian products of involutive graphs and prove Theorem 1.5. The class of involutive graphs has been introduced in [11]. It contains graphs like even cycles, hypercubes or more generally diametrical graphs and even graphs (see [9]). Let us recall the definition. An involutive graph $H$ is a graph such that there exists an involution, bar : $V(H) \rightarrow V(H)$, which commutes with all automorphisms and has no fixed point. In other words:

- for every $u \in V(H)$, we have $\overline{\bar{u}}=u$ and $\bar{u} \neq u$,

- for every $\sigma \in \operatorname{Aut}(H)$ and every $u \in V(H)$, we have $\sigma(\bar{u})=\overline{\sigma(u)}$.

The set $\{u, \bar{u}\}$ will be called a block. An important remark is that an automorphism of an involutive graph has to map a block to a block. In other words, there is a natural action of the automorphism group on the set of blocks. We introduce the following concepts, which are going to play a similar role as the meta-colors used in Section 3.

If $H$ is an involutive graph and $c$ is a vertex-coloring with $d$ colors, then the type $t$ of a block $\{u, \bar{u}\}$ is defined by: 


$$
t(\{u, \bar{u}\})= \begin{cases}c(u)-c(\bar{u}) \bmod d & \text { if } c(u)-c(\bar{u}) \bmod d \in\left\{0, \ldots,\left\lfloor\frac{d}{2}\right\rfloor\right\} \\ c(\bar{u})-c(u) \bmod d & \text { otherwise. }\end{cases}
$$

The block-list of $H, L_{c}(H)$ is the list $\left(n_{0}, \ldots, n_{\left\lfloor\frac{d}{2}\right\rfloor}\right)$ of length $\left\lfloor\frac{d}{2}\right\rfloor+1$, where $n_{i}$, with $i \in\left\{0, \ldots,\left\lfloor\frac{d}{2}\right\rfloor\right\}$, is the number of blocks of type $i$, according to the coloring $c$. Note that, if $\sigma$ is an automorphism of $H$, then $t(\sigma(\{u, \bar{u}\}))=t(\{u, \bar{u}\})$ and $L_{c}(\sigma(H))=L_{c}(H)$.

Assume now that $H$ is a connected involutive graph and $F$ is a connected graph relatively prime to $H$. The following proposition asserts that if the classical distinguishing number of $F$ is not too big, then $D_{\mathcal{R}}(H \square F)$ is bounded above by $D_{\mathcal{R}}(H)$. Theorem 1.5 will be a straightforward application of this result.

Theorem 4.1. Let $H$ be a connected involutive graph. Assume that Gentle has a winning strategy playing second on $H$, with $d \geq D_{\mathcal{R}}(H)$ colors. Moreover, this strategy yields colorings, whose block-list is in a fixed set $\mathcal{L}$.

If $F$ is a connected graph relatively prime to $H$, with $D(F) \leq\left(\begin{array}{c}\frac{|V(H)|}{2}+\left\lfloor\frac{d}{2}\right\rfloor \\ \left\lfloor\frac{d}{2}\right\rfloor\end{array}\right)-|\mathcal{L}|+1$, then $D_{\mathcal{R}}(H \square F) \leq d$.

Proof. We have to give a Gentle winning strategy with $d$ colors, assuming Rascal starts. The coloring obtained at the end of the game will be denoted by $c$.

First of all, Gentle will follow an $H$-fiber strategy. Note that $|V(H)|$ is even. Hence, we are in Case 0 of this strategy. Let $\left(u_{1}, v_{1}\right)$ be the first vertex of $H \square F$ colored by Rascal. Gentle imagines a distinguishing coloring $c^{\prime}$ of $F$ with $D(F)$ colors. When Gentle has to play in the fiber $H^{v_{1}}$, he chooses the vertex and the color according to a winning strategy in $H$. This is possible, because Gentle's moves and Rascal's moves in $H^{v_{1}}$ alternate like the moves in a game played only on $H$, when Rascal starts (see Proposition 2.1). Moreover, $d \geq D_{\mathcal{R}}(H)$ by hypothesis. In the other $H$-fibers, when Rascal plays the vertex $(u, v)$, Gentle answers by coloring the vertex $(\bar{u}, v)$. In this way, Gentle will be able to control the block-list of these fibers. More precisely, he chooses the colors such that:

$$
(\dagger) \text { for every } v, w \in V(F), L_{c}\left(H^{v}\right)=L_{c}\left(H^{w}\right) \text { if } c^{\prime}(v)=c^{\prime}(w) .
$$

This is possible if there exists at least $(D(F)-1)+|\mathcal{L}|$ distinct possible block-lists. Indeed, Gentle cannot control in advance the block-list of the fiber $H^{v_{1}}$. By hypothesis, we only know that this block-list will belong to $\mathcal{L}$. Hence, $|\mathcal{L}|$ kinds of block-list are used to stand for the imaginary color $c^{\prime}\left(v_{1}\right)$. Finally, with $(D(F)-1)+|\mathcal{L}|$ possible blocklists, Gentle has enough possibilities to associate distinct block-lists to distinct colors of the coloring $c^{\prime}$. The number of block-lists is the number of weak compositions of $\frac{|V(H)|}{2}$ (the number of blocks) into $\left\lfloor\frac{d}{2}\right\rfloor+1$ natural numbers (the number of block types). So,

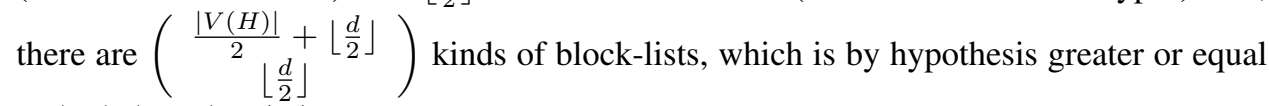
to $(D(F)-1)+|\mathcal{L}|$.

Now, we prove that the coloring obtained with this strategy is distinguishing. Assume $\sigma$ is a color preserving automorphism. We have $\sigma=(\psi, \phi)$, where $\psi \in \operatorname{Aut}(H)$ and $\phi \in \operatorname{Aut}(F)$. This automorphism maps blocks in $H^{v}$ to blocks in $H^{\phi(v)}$, for any $v \in$ $V(F)$. Hence, the automorphism $\phi$ preserves the block-lists of the $H$-fibers: $L_{c}\left(H^{v}\right)=$ $L_{c}\left(H^{\phi(v)}\right)$, for all $v \in V(F)$. By condition $(\dagger)$, this automorphism preserves also the 
distinguishing coloring $c^{\prime}$ of $F$. Hence, $\phi$ is the identity of $\operatorname{Aut}(F)$. This implies that $\sigma\left(H^{v_{1}}\right)=H^{v_{1}}$. But the coloring of this $H$-fiber is obtained by following a winning strategy for Gentle in the game on $H$. Therefore, $\psi$ is the identity of $H$. In conclusion, $\sigma$ is trivial and the coloring $c$ is distinguishing.

Theorem 1.5 is a straightforward application of the above result for two reasons. First, we know that for an involutive graph $H$, we have $D_{\mathcal{R}}(H) \leq D^{2}(H)+D(H)-2$ (see Theorem 1.3). Moreover, with $D^{2}(H)+D(H)-2$ colors, Gentle has a winning strategy such that he knows exactly the block-list he will get at the end of the game (see the proof of Theorem 1.6 in [11]). It means, with the notation of the above theorem, that $\mathcal{L}$ is just a singleton.

\section{Cartesian products of cycles}

In this final section, we give a proof of Theorem 1.6. Note that proving the statement about the infinity of the invariants is a straightforward application of Proposition 1.1. For the cycle $C_{n}$ of order $n \geq 3$, we set $V\left(C_{n}\right)=\{1, \ldots, n\}$ and $E\left(C_{n}\right)=\{i j|| i-j \mid=$ $\left.1 \bmod n, i, j \in V\left(C_{n}\right)\right\}$. We begin with toroidal grids of even order. Since even cycles are involutive graphs the first proposition is a direct corollary of Theorem 4.1.

Proposition 5.1. Let $n$ and $m$ be two distinct natural numbers greater or equal to 3 . If $n$ is even and $n \geq 8$, then $D_{\mathcal{R}}\left(C_{n} \square C_{m}\right)=2$.

Proof. In [11, Proposition 4.1], the winning strategy used by Gentle with two colors leads to exactly three bi-chromatic blocks, when $n \geq 12$. To show that $D_{\mathcal{R}}\left(C_{8}\right)=D_{\mathcal{R}}\left(C_{10}\right)=$ 2 , they used an exhaustive computer check. This computing also gives that there is a Gentle's winning strategy which leads to one or three bi-chromatic blocks if $n=8$, and to one or four bi-chromatic blocks if $n=10$. Therefore, with the same notation as in Theorem 4.1, we have $|\mathcal{L}| \leq 2$. For all $m \geq 3$, we have $D\left(C_{m}\right) \leq 3$. Thus, we have $D\left(C_{m}\right) \leq\left(\begin{array}{c}\frac{n+2}{2} \\ 1\end{array}\right)-2+1$, and we can directly apply Theorem 4.1 to get $D_{\mathcal{R}}\left(C_{n} \square C_{m}\right)=2$.

Proposition 5.2. Let $n$ be in $\{4,6\}$ and $F$ a connected graph relatively prime to $C_{n}$, with at least three vertices. If $D(F) \leq 3$, then $D_{\mathcal{R}}\left(C_{n} \square F\right)=2$.

Proof. Let $n \in\{4,6\}$. We denote by $c$ the coloring built during the game. We have to give a winning strategy for Gentle with 2 colors. Gentle plays according to a $C_{n}$-fiber strategy and uses the block-lists as meta-colors. Here, the problem is that $D_{\mathcal{R}}\left(C_{n}\right)=3$. Gentle fancies a distinguishing coloring $c^{\prime}$ of $F$, where the three colors are really used. As in Theorem 4.1, Gentle can control the block-list of the $C_{n}$-fibers such that:

$$
\text { for every } v \in V(F), L_{c}\left(C_{n}^{v}\right)= \begin{cases}(n, 0) & \text { if } c^{\prime}(v)=1 \\ (n-1,1) & \text { if } c^{\prime}(v)=2, \\ (n-2,2) & \text { if } c^{\prime}(v)=3\end{cases}
$$

Moreover, for $v \in V(F)$, if $L_{c}\left(C_{n}^{v}\right)$ must be equal to $(n-1,1)$ or $(n-2,2)$, he plays such that the block $\{(1, v),(n / 2, v\}$ is of type 1 .

Now, we prove that the coloring $c$ is distinguishing. Assume $\sigma$ is a color preserving automorphism. We have $\sigma=(\psi, \phi)$, where $\psi \in \operatorname{Aut}\left(C_{n}\right)$ and $\phi \in \operatorname{Aut}(F)$. For all 
$v \in V(F), L_{c}\left(C_{n}^{v}\right)=L_{c}\left(C_{n}^{\phi(v)}\right)$. Hence, for all $v \in V(F), c^{\prime}(v)=c^{\prime}(\phi(v))$. Since $c^{\prime}$ is a distinguishing coloring of $F$, we get that $\phi$ is trivial. Hence, $\sigma$ fixes the $C_{n}$-fibers setwise. Since there is at least one $C_{n}$-fiber with block-list $(n-1,1), \psi$ must be the symmetry $\Delta$ of axes $\left(1, \frac{n}{2}\right)$ or the identity. But $\Delta$ does not preserve the coloring in the $C_{n}$-fibers, whose block-list is $(n-2,2)$. Indeed, in such a fiber, one of the block of type 1 is stable under $\Delta$. The other block of type 1 is sent by $\Delta$ to a block of type 0 or switched to itself. In both cases, it breaks the coloring. In conclusion, $\psi$ is the identity and so is $\sigma$.

This result directly implies the following corollary.

Corollary 5.3. Let $m$ be an integer greater or equal to 3 .

1. If $m \neq 6$, then $D_{\mathcal{R}}\left(C_{6} \square C_{m}\right)=2$.

2. If $m \neq 4$, then $D_{\mathcal{R}}\left(C_{4} \square C_{m}\right)=2$.

The following proposition gives a general upper bound, when one factor has distinguishing number less or equal to 2 . A corollary of this result is the case where both factors have odd cardinality and at least one of them is not prime.

Proposition 5.4. Let $H$ and $F$ be two connected relatively prime graphs. Assume $H$ is vertex transitive, $D(H) \geq 2$ and $D(F) \leq 2$.

1. If $|V(H)|$ and $|V(F)|$ are odd, then $D_{\mathcal{G}}(H \square F) \leq D_{\mathcal{G}}(H)$.

2. If $|V(H)|$ is odd and $|V(F)|$ is even, then $D_{\mathcal{R}}(H \square F) \leq D_{\mathcal{G}}(H)$.

3. If $|V(H)|$ is even, then $D_{\mathcal{R}}(H \square F) \leq D_{\mathcal{R}}(H)$.

Proof. We prove the first statement. Let $c$ be the coloring built during the game. For each $H$-fiber $H^{v}$, with $v \in V(F)$, we define:

$$
p\left(H^{v}\right)= \begin{cases}1 & \text { if }\left|\left\{u \in H^{v} \mid c((u, v))=1\right\}\right| \text { is odd } \\ 2 & \text { otherwise. }\end{cases}
$$

We have to define a Gentle's winning strategy with $D_{\mathcal{G}}(H)$ colors. Gentle is going to play according to an $H$-fiber strategy. Note that we are in Case 1 of this strategy. In the $H$ fibers, where Gentle is the first to play, the moves alternate exactly as in a game played only on $H$, with Gentle playing first (see Proposition 2.1). In the other $H$-fibers, it is also the case, except for the first move which is played by Rascal. In other words, Rascal will play the two first moves in a row in these $H$-fibers. Since $H$ is vertex transitive, we assume, by Lemma 2.2, that Gentle has played first also in these $H$-fibers. Therefore, Gentle can play following a winning strategy for $H$ in each $H$-fiber. He plays like this as long as one $H$-fiber is totally colored, say $H^{v_{0}}$. Now, he fancies a distinguishing coloring $c^{\prime}$ of $F$ such that $c^{\prime}\left(v_{0}\right)=p\left(H^{v_{0}}\right)$. For the later moves, he plays such that:

$(\ddagger)$ for every $v \in V(F), c^{\prime}(v)=p\left(H^{v}\right)$.

Since he follows an $H$-fiber strategy, we recall that he is going to play the last move in each $H$-fiber. Hence, he is able to decide the parity of the number of vertices colored with 1 in each of them. 
Let us prove now that the coloring $c$ is distinguishing. Let $\sigma$ be a color preserving automorphism. For all $v \in V(F)$, we have $p\left(\sigma\left(H^{v}\right)\right)=p\left(H^{v}\right)$. Since $c^{\prime}$ is a distinguishing coloring of $F$, it implies, by $(\ddagger)$, that $\sigma$ fixes the $H$-fibers setwise. Therefore, $\sigma\left(H^{v_{0}}\right)=$ $H^{v_{0}}$. But the coloring on this $H$-fiber is obtained by following a winning strategy on $H$. Then, $H^{v_{0}}$ must be fixed pointwise by $\sigma$. In conclusion, $\sigma$ is the identity.

For the two remaining statements, the proof is almost the same. The only difference is that for the third item, we are in Case 0 of the $H$-fiber strategy.

Corollary 5.5. Let $n$ and $m$ be two distinct odd natural numbers greater or equal to 3 . If $n$ is not prime and $m \geq 7$, then $D_{\mathcal{G}}\left(C_{n} \square C_{m}\right)=2$.

Proof. Under the hypothesis of the corollary, we have $D_{\mathcal{G}}\left(C_{n}\right)=2$ and $D\left(C_{m}\right)=2$. Thus, this is a straightforward application of Proposition 5.4.

With the previous results, we are able to compute the distinguishing numbers of the toroidal grid $C_{n} \square C_{m}$, except for the following cases:

- $C_{3} \square C_{m}$, with $m \neq 3$ and $m$ odd,

- $C_{5} \square C_{m}$, with $m \neq 5$ and $m$ odd,

- $C_{n} \square C_{m}$, with $n \neq m, n$ and $m$ odd and prime.

To settle this remaining cases, we state the following proposition.

Proposition 5.6. Let $n$ and $m$ be two two distinct odd natural numbers greater or equal to 3. If $n$ is prime and $m \geq 7$, then $D_{\mathcal{G}}\left(C_{n} \square C_{m}\right)=2$.

Proof. Let $c$ be the coloring built during the game. For each $C_{n}$-fiber $C_{n}^{j}$, with $j \in$ $\{1, \ldots, m\}$, we define:

$$
p\left(C_{n}^{j}\right)= \begin{cases}1 & \text { if }\left|\left\{i \in C_{n}^{j} \mid c((i, j))=1\right\}\right| \text { is odd } \\ 2 & \text { otherwise. }\end{cases}
$$

Let $M_{1}, M_{2}$ and $M_{3}$ be three maximum matchings of $C_{n}$, whose union covers $E\left(C_{n}\right)$. Let $v_{1}, v_{2}$ and $v_{3}$ be the only vertices of $C_{n}$, which are respectively not covered by $M_{1}, M_{2}$ and $M_{3}$. Let $c^{\prime}$ be a distinguishing coloring of $C_{m}$, with 2 colors. Such a coloring exists because $m>5$.

We have to outline a Gentle's winning strategy with 2 colors. He is going to follow a $C_{n}$-fiber strategy. Since $m \geq 7$, there are at least three distinct $C_{n}$-fibers, $C_{n}^{j_{1}}, C_{n}^{j_{2}}, C_{n}^{j_{3}}$, with $j_{1}, j_{2}, j_{3} \in\{1, \ldots, m\}$, where Gentle is the first to play. The first vertex that Gentle is going to color in $C_{n}^{j_{k}}$, with $k \in\{1,2,3\}$ is $\left(v_{k}, j_{k}\right)$. He colors it such that $c\left(\left(v_{k}, j_{k}\right)\right)=$ $c^{\prime}\left(j_{k}\right)$. In the other $C_{n}$-fibers, where he is the first to play, the first vertex he chooses and the color he uses do not matter. For the later moves in $C_{n}^{j_{k}}$, he will choose the vertices with respect to the matching $M_{k}$. Moreover, he uses a color distinct from the one used by Rascal just before. In this way, the parity of the number of vertices in $C_{n}^{j_{k}}$ colored with 1 only depends on $c\left(\left(v_{k}, j_{k}\right)\right)$. Hence $p\left(C_{n}^{j_{k}}\right)=c\left(\left(v_{k}, j_{k}\right)\right)=c^{\prime}\left(j_{k}\right)$, for $k \in\{1,2,3\}$. For the moves in $C_{n}^{j}$, with $j \notin\left\{j_{1}, j_{2}, j_{3}\right\}$, Gentle plays whatever he wants, except when he colors the last vertex of the $C_{n}$-fiber. In that case, he chooses the color such that $p\left(C_{n}^{j}\right)=c^{\prime}(j)$.

We prove now that $c$ is distinguishing. Let $\sigma$ be a color preserving automorphism. For all the $C_{n}$-fibers, we have $p\left(C_{n}^{j}\right)=c^{\prime}(j)$. Since $c^{\prime}$ is a distinguishing coloring of 
$C_{m}$, the automorphism $\sigma$ fixes the $C_{n}$-fibers setwise. Thus, we have $\sigma=(\psi, \mathrm{id})$, with $\psi \in \operatorname{Aut}\left(C_{n}\right)$. Since $n$ is prime, any nontrivial rotation acts transitively on a $C_{n}$-fiber. As at least one such fiber is not monochromatic, $\psi$ could not be a nontrivial rotation. On the other hand, if $\psi$ is an axial symmetry, since $n$ is odd, there is an edge $e \in E\left(C_{n}\right)$ such that $\psi(e)=e$. This edge belongs to one of the three matchings, say $M_{1}$. Gentle has played such that in $C_{n}^{j_{1}}$, the edge corresponding to $e$ is not monochromatic. Therefore $\psi$ cannot preserve the coloring. In conclusion, $\psi$ must be the identity and so is $\sigma$.

We are now ready to prove Theorem 1.6.

Proof of Theorem 1.6. Let $n_{1}, \ldots, n_{k}$, with $k \geq 2$, be $k$ distinct numbers greater or equal to 3 . If $\prod_{i=1}^{k} n_{i}$ is even (resp. odd), we have to prove that $D_{\mathcal{R}}\left(C_{n_{1}} \square \cdots \square C_{n_{k}}\right)=2$ (resp. $D_{\mathcal{G}}\left(C_{n_{1}} \square \cdots \square C_{n_{k}}\right)=2$ ). If $k=2$, this is a consequence of Propositions 5.1 and 5.6 and Corollaries 5.3 and 5.5, except for $C_{3} \square C_{5}$. An exhaustive computer check proves that in that case two colors are also enough. For $k \geq 3$, we proceed by induction. If we are not dealing with $C_{3} \square C_{4} \square C_{5}$, we can assume that $n_{k} \geq 6$. Hence, $D\left(C_{n_{k}}\right)=2$ and by induction $D_{\mathcal{R}}\left(C_{n_{1}} \square \cdots \square C_{n_{k-1}}\right)=2$ or $D_{\mathcal{G}}\left(C_{n_{1}} \square \cdots \square C_{n_{k-1}}\right)=2$, depending on the parity. Finally, we apply Proposition 5.4, with $H=C_{n_{1}} \square \cdots \square C_{n_{k-1}}$ and $F=$ $C_{n_{k}}$, to get the expected results. For $C_{3} \square C_{4} \square C_{5}$, we apply Proposition 5.2 to show that $D_{\mathcal{R}}\left(C_{3} \square C_{4} \square C_{5}\right)=2$.

Corollary 5.7. Let $n_{1}, \ldots, n_{k}$ be $k$ distinct natural numbers greater or equal to 2 .

1. If $\prod_{i=1}^{k} n_{i}$ is even, then $D_{\mathcal{G}}\left(P_{n_{1}} \square \cdots \square P_{n_{k}}\right)=\infty$ and $D_{\mathcal{R}}\left(P_{n_{1}} \square \cdots \square P_{n_{k}}\right)=2$.

2. If $\prod_{i=1}^{k} n_{i}$ is odd, then $D_{\mathcal{R}}\left(P_{n_{1}} \square \cdots \square P_{n_{k}}\right)=\infty$ and $D_{\mathcal{G}}\left(P_{n_{1}} \square \cdots \square P_{n_{k}}\right)=2$.

Proof. If $k=1$, we easily have $D_{\mathcal{R}}\left(P_{n}\right)=2$, when $n$ is even and $D_{\mathcal{G}}\left(P_{n}\right)=2$, when $n$ is odd (see [11]). If $k \geq 2$ and $n_{i} \geq 3$, for all $i \in\{1, \ldots, k\}$, then it is a straightforward consequence of Theorem 1.6. Indeed, in this case, a distinguishing coloring of $C_{n_{1}} \square \cdots \square C_{n_{k}}$ is also a distinguishing coloring of $P_{n_{1}} \square \cdots \square P_{n_{k}}$. If one factor, say $P_{n_{1}}$ is isomorphic to $P_{2}$, then we can apply Proposition 5.4, with $H=P_{n_{1}}$ and $F=P_{n_{2}} \square \cdots \square P_{n_{k}}$. Hence, $D\left(P_{n_{2}} \square \cdots \square P_{n_{k}}\right)=2$ (the only Cartesian products of paths for which it is not true are $P_{2} \square P_{2}$ and $P_{2} \square P_{2} \square P_{2}$, with $\left.D\left(P_{2} \square P_{2}\right)=D\left(P_{2} \square P_{2} \square P_{2}\right)=3\right)$.

\section{Acknowledgements}

The research was in part financed by the ANR-14-CE25-0006 project of the French National Research Agency.

\section{References}

[1] M.O. Albertson and K.L. Collins, Symmetry breaking in graphs, Electron. J. Comb. 3 (1996), \#R18.

[2] B. Bogstad and L. Cowen, The distinguishing number of hypercubes, Discrete Math. 383 (2004), 29-35, doi: 10.1016/j.disc.2003.11.018. 
[3] B. Brešar, S. Klavžar and D.F. Rall, Domination game and an imagination strategy, SIAM J. Discrete Math. 24 (2010), 979-991, doi: 10.1137/100786800.

[4] I. Broere and M. Pilśniak, The distinguishing index of the Cartesian product of countable graphs, Ars Math. Contemp. 13 (2017), 15-21.

[5] E. Estaji, W. Imrich, R. Kalinowski, M. Pilśniak and T. Tucker, Distinguishing Cartesian products of countable graphs, Discuss. Math. Graph Theory 37 (2017), doi:10.7151/dmgt.1902.

[6] U. Faigle, U. Kern, H. Kierstead and W.T. Trotter, On the game chromatic number of some classes of graphs, Ars Combinatoria 35 (1993), 143-150.

[7] M.J. Fisher and G. Isaak, Distinguishing colorings of Cartesian products of complete graphs, Discrete Math. 308 (2008), 2240-2246, doi: 10.1016/j.disc.2007.04.070.

[8] M.J. Fisher and G. Isaak, Distinguishing numbers of Cartesian products of multiple complete graphs, Ars Math. Contemp. 5 (2012), 159-173.

[9] F. Göbel and H.J. Veldman, Even graphs, J. Graph Theory 10 (1986), 225-239, doi: 10.1002/jgt.3190100212.

[10] A. Gorzkowska, R. Kalinowski and M. Pilśniak, The distinguishing index of the Cartesian product of finite graphs, Ars Math. Contemp. 12 (2017), 77-87.

[11] S. Gravier, K. Meslem, S. Schmidt and S. Slimani, A new game invariant of graphs: the game distinguishing number, Discrete Math. Theor. Comput. Sci. 19 (2017).

[12] W. Imrich, J. Jerebic and S. Klavžar, The distinguishing number of Cartesian products of complete graphs, Eur. J. Comb. 45 (2008), 922-929, doi: 10.1016/j.ejc.2007.11.018.

[13] W. Imrich and S. Klavžar, Distinguishing Cartesian powers of graphs, J. Graph Theory 53 (2006), 250-260, doi: 10.1002/jgt.20190.

[14] W. Imrich and S. Klavžar, Product graphs: structure and recognition, Wiley-Interscience Series in Discrete Mathematics and Optimization, 2000.

[15] S. Klavžar, T.L. Wong and X. Zhu, Distinguishing labellings of group action on vector spaces and graphs, J. Algebra 15 (2) (2006), 626-641, doi: 10.1016/j.jalgebra.2006.01.045.

[16] S. Klavžar and X. Zhu, Cartesian powers of graphs can be distinguished by two labels, Eur. J. Comb. 28 (2007), 303-310, doi: 10.1016/j.ejc.2005.07.001. 